\title{
IMAGE SEGMENTATION USING PARALLEL SELF ORGANIZING TREE MAP
}

\author{
Xiaoming Fan ${ }^{1}$, Jonathan Randall ${ }^{2}$, Ivan Lee \\ ${ }^{l}$ Department of Electrical and Computer Engineering \\ Ryerson University, Toronto, Canada \\ ${ }^{2}$ School of Electrical and Information Engineering \\ The University of Sydney, NSW, Australia
}

\begin{abstract}
At present, the researchers are making great effort to search for a general purpose resolution for image segmentation. Most of the approaches we are using now are somewhat inefficient and tardy. In this paper we present an innovative method, Parallel Self-organizing Tree Map (PSOTM), which is improved from SOTM. By processing image in parallel, PSOTM could segment the image in a much faster processing speed. After applying the new technology, PSOTM, we could obtain the higher efficiency with limited impacts on the visual quality.
\end{abstract}

\section{INTRODUCTION}

Currently, there are two key topics in the image field, one is image segmentation and the other is image recognition. The researchers have taken great efforts to develop new technologies for image segmentation and recognition [1][2][3]. In early years, most image segment projects only could be done by human being. However, the high expense and low efficiency of handwork are always the issues in image processing. How to classify a picture into different segments intelligently and how to improve the efficiency of segmentation software synchronously are two most popular issues. Recent research makes the suitable self-driven image segmentation technologies will be available in the near future.

Self Organizing Tree Map (SOTM) [4] is a special algorithm derived from Self-Organization Map (SOM) with tree building hierarchy, thus improving the accuracy for determining the correct number of centroids automatically. SOTM can be seen as a mapping from a high dimensional Euclidean space onto a finite set of prototypes. SOTM has been successfully applied for image restoration, compression, and segmentation [5]. Furthermore, the computing method, SOTM, is used to attain not only the feature points, but also the center vectors of feature points. It has proved its classification abilities in performing image segmentation and feature point grouping in the image processing.
The novel approach has proved to be a potential method to minimize the human parts in image processing and automatically segmenting the images. Nevertheless, the deficiency coming with SOTM is also distinctly, it is still a time-consuming approach. In addition, the hardware and software requirement of the method are generally critical and relatively exorbitant. How can we improve the efficiency of the SOTM while retaining its cost?

In the paper, we propose "Parallel Self-organizing Tree Map (PSOTM)", which aims to improve the processing speed of SOTM. It uses parallel processes to provide a quick segmentation in image processing. Unlike SOTM, at the beginning of each job, PSOTM divides the image into several parts and assigns the different parts into multiple SOTM processes in parallel, and each process only deals with a part of the image. At the end of the procedure, PSOTM analyzes all the results from different processes and updates the final center points.

\section{IMAGE SEGMENTATION WITH SOTM AND PARALLEL SOTM}

There are two stages in the learning rule of SOTM, one is locating stage and the other is convergence stage. The learning rate is controlled by adaptation parameter $\alpha(t)$ [4][5], which decreases with time of weight vectors approach the group centers. It shows with a linear function or an exponential function. It is not difficult to see that adaptation parameter $\alpha(t)$ just gives a rough weight value, could not represent the number of the feature points that has been processed; on the contrary, in PSOTM we weight each center point with size of its cluster points instead of adaptation parameter $\alpha(t)$. It could represent the weight much more precisely than adaptation parameter of SOTM.

In this paper, the features extracted from the raw image file include the Luminance and Chrominance components in HSV domain, and the same values of the image undertaking low-pass filters using $3 \times 3$ window blocks. Thus, a sixdimension vector is generated for each pixel in the image. To reduce the dimension of the input data, these vectors are pre-processed using Principle Component Analysis (PCA) [6]. PCA has proved to be a useful statistical technique in 
face recognition and image compression fields. The variance in the principle components also could be captured by PCA. Thus, the three-dimensional vectors regenerated from PCA are served as the input data for the PSOTM process.

Figure 1 shows the PSOTM image processing procedure. In the Initial Stage, PSOTM analyzes the image, and generates the feature points. In Stage 1, PSOTM assigns feature points into different processes. In Stage 2, SOTM processes feature points in each process, obtains the center points. In Stage 3, PSOTM joins the center points from all the processes. Finally in Stage 4, PSOTM reprocesses the center points, produces final center points.

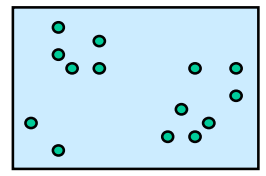

Initial Stage
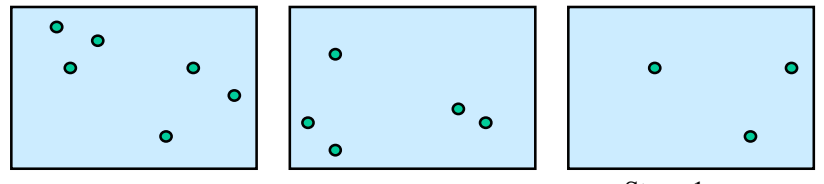

Stage 1
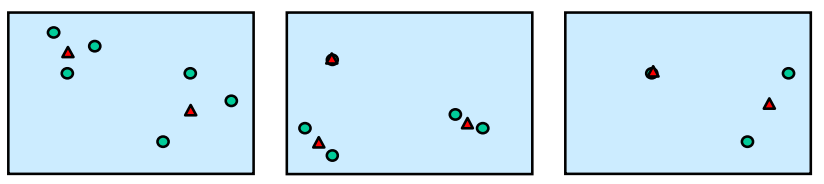

Stage 2

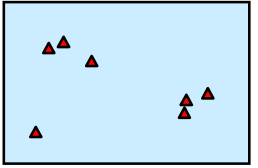

Stage 3

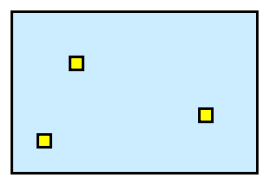

Stage 4

- feature point $\Delta$ center point $\square$ final center point

Figure 1 Parallel SOTM procedure

In Stage 1-2, the neuron update process has been modified and be denoted in equation (1). Let $c(n)$ denotes the center point of first $n$ feature points, $c(n+1)$ denotes the center point of $(n+1)$ feature points and $v(n+1)$ denotes the $(n+1)$ th feature point. Then, $c(n+1)$ is updated by $c(n)$ and $v(n+1)$ in equation (1).

$$
\begin{aligned}
c(n+1) & =\frac{c(n) \cdot n+v(n+1)}{n+1} \\
& =c(n)+\frac{1}{n+1}[v(n+1)-c(n)]
\end{aligned}
$$

In Stage 3-4, the update process could be regarded as weighted SOTM and denotes in equation (2). Let $c(n+1)$ denotes the $(n+1)$ th center point, csize $(i)$ denotes the size of $i$ th center point, csize $(n+1)$ denotes the size of $(n+1)$ th center point, $f(n)$ denotes the final center point of first $n$ center points and $f(n+1)$ denotes the final center point of $(n+1)$ center points. Therefore, the equation (2) shows the $f(n+1)$ is the center of weighted $f(n)$ and weighted $c(n+1)$.

$$
\begin{aligned}
f(n+1) & =\frac{f(n) \cdot \sum_{i=1}^{n} c_{\text {size }}(i)+c(n+1) \cdot c_{\text {size }}(n+1)}{\sum_{i=1}^{n+1} c_{\text {size }}(i)} \\
& =f(n)+\frac{c(n+1) \cdot c_{\text {size }}(n+1)-f(n) \cdot c_{\text {size }}(n+1)}{\sum_{i=1}^{n+1} c_{\text {size }}(i)} \\
& =f(n)+\frac{c_{\text {size }}(n+1)}{\sum_{i=1}^{n+1} c_{\text {size }}(i)}[c(n+1)-f(n)]
\end{aligned}
$$

The same algorithm described above can be used for SOTM by setting $c$ size $(i)=1$ in equation (2), which can be simplified as shown in equation (3).

$$
\begin{aligned}
f(n+1) & =\frac{f(n) \cdot \sum_{i=1}^{n} c_{\text {size }}(i)+c(n+1) \cdot c_{\text {size }}(n+1)}{\sum_{i=1}^{n+1} c_{\text {size }}(i)} \\
& =\frac{f(n) \cdot n+c(n+1)}{n+1}
\end{aligned}
$$

The whole process of PSOTM could be interpreted by below pseudo code.

Let $d(x, y)$ denotes the Euclidean distance between point $\mathrm{x}$ and $\mathrm{y}$. Let $H(t)$ denotes the hierarchy control function at iteration $\mathrm{t}$, which determines whether a current centroid will be updated or a new centroid will be created.

In Figure 2, we show the pseudo code of PSOTM procedure. The code is mainly made up with one FOR and three IF loops. 


\begin{tabular}{|l|}
\hline FOR each root node of all centroid nodes \\
IF $\operatorname{Min}(w)>d($ data point $x$,centroid $)$ \\
THEN $M i n(w)=d($ data point $x$, centroid $)$ \\
END IF \\
IF $d(x$,centroid $)<H(t)$ \\
THEN \\
winning centroid $=$ the reinforced learning rule \\
ELSE \\
new centroid node $=x$ \\
INCREMENT Count \\
ENDIF \\
IF Max $(d(x$, centroid $))>$ max_distance \\
Or iteration $>$ max_iteration \\
or centroid $>$ max_centroid \\
THEN \\
Exit \\
ENDIF \\
End FOR
\end{tabular}

Figure 2 Pseudo code of PSOTM

\section{ERROR MEASUREMENT}

Let $\backslash$ and $\|\cdot\|$ denote set difference and cardinality, respectively. Let $R(S, p i)$ represent the set of pixels corresponding to the region in segmentation $S$ that contains pixel $p i$, the local refinement error is defined by

$\operatorname{LRE}\left(S_{1}, S_{2}, p_{i}\right)=\frac{\left\|R\left(S_{1}, p_{i}\right) \backslash R\left(S_{2}, p_{i}\right)\right\|}{\left\|R\left(S_{1}, p_{i}\right)\right\|}$

The Global Consistency Error $(G C E)$ and Local Consistency Error ( $L C E)$ are defined by

$$
\begin{aligned}
& \operatorname{GCE}\left(S_{1}, S_{2}\right)=\frac{1}{n} \min \left\{\sum_{i} \operatorname{LRE}\left(S_{1}, S_{2}, p_{i}\right), \sum_{i} \operatorname{LRE}\left(S_{2}, S_{1}, p_{i}\right)\right\} \\
& \operatorname{LCE}\left(S_{1}, S_{2}\right)=\frac{1}{n} \sum_{i} \min \left\{\operatorname{LRE}\left(S_{1}, S_{2}, p_{i}\right), \operatorname{LRE}\left(S_{2}, S_{1}, p_{i}\right)\right\}
\end{aligned}
$$

\section{EXPERIMENT}

In the paper, we investigate five setups, $\operatorname{SOTM}(\mathrm{N}=1)$ and Parallel SOTM ( $=2$ to 5) respectively. After the image has been segmented by the system, we compare the results of each PSOTM $(\mathrm{N}=2$ to 5$)$ with the result of SOTM $(\mathrm{N}=1)$. The error measurement of the results is characterized in terms of GCE and LCE.

Figure 3 shows the segmentation results of the house image. The first picture, Figure 3(a) is the original picture, Figure $3(\mathrm{~b})$ is generated by SOTM and Figure 3(c) is produced by PSOTM $(\mathrm{N}=5)$. Comparing with original image, Figure 3(b), which processed by SOTM, has become less detail, but clear-cut. It took around one day to finish the process. The visual quality of Figure 3(c), which is generated by PSOTM, is comparable with Figure 3(b). The walls of the house, the trees around house and the shadow of the house are almost the same. In the experiment, the effect of parallel SOTM on the image quality is limited. However, the difference between Figure 3(b) and Figure 3 (c) is also existed. Several detail points only show in Figure 3(c) and the frames of the windows in Figure 3(c) is much clearer than Figure 3(b).

In the Table 1, GCE and LCE values of $\mathrm{N}(\mathrm{N}=2,3,4$, 5) Parallel SOTM are in the same level. The increments of GCE and LCE values are not proportional to the number of processes in the PSOTM. That means no matter how many processes we used in the PSOTM, the results are always in the same range. Sometimes, we could even get much more precise results in less time by increasing the number of processes.

A ping-pong player picture was used in the second experiment. For the purpose of the comparison, Figure 4(a) is the original picture. 4(b) and 4(c) are results generated by SOTM and PSOTM respectively. Since the original picture we used in the experiment has less detail and much simple content. We could not tell too much difference between the results of SOTM and PSOTM. The GCE and LCE values of $\mathrm{N}(\mathrm{N}=2,3,4,5)$ parallel SOTM are also showing in the Table 1. Consequently, it is easy to conclude that the error ranges between the results of different parallel processes and SOTM are in the same level.

Table 1 Segmentation performance with different number of

\begin{tabular}{|l|l|l|l|l|}
\hline & $\mathrm{N}=2$ & $\mathrm{~N}=3$ & $\mathrm{~N}=4$ & $\mathrm{~N}=5$ \\
\hline $\begin{array}{l}\text { LCE } \\
\text { (house) }\end{array}$ & 0.15 & 0.15 & 0.1 & 0.18 \\
\hline $\begin{array}{l}\text { GCE } \\
\text { (house) }\end{array}$ & 0.16 & 0.16 & 0.12 & 0.2 \\
\hline $\begin{array}{l}\text { LCE } \\
\text { (Ping-Pong) }\end{array}$ & 0.0124 & 0.0221 & 0.0340 & 0.0325 \\
\hline $\begin{array}{l}\text { GCE (Ping-Pong) } \\
\text { (PCE }\end{array}$ & 0.0170 & 0.0291 & 0.0408 & 0.0356 \\
\hline
\end{tabular}

PSOTM processes

\section{DISCUSSION ON PERFORMANCE IMPROVEMENT}

In the experiment, PSOTM assigns all feature points into $\mathrm{N}$ (2-5) processes. Therefore, it likes concurrently N SOTMs are processing one image. As a result, the computer finishes the process in approximate one Nth of original time. From the comparison between the results of different PSOTMs, we discover that the error measurements of results are in the same level. Thus, it proves the PSOTM could significantly improve the processing efficiency with almost the same results. In conclusion, the PSOTM makes it possible to 


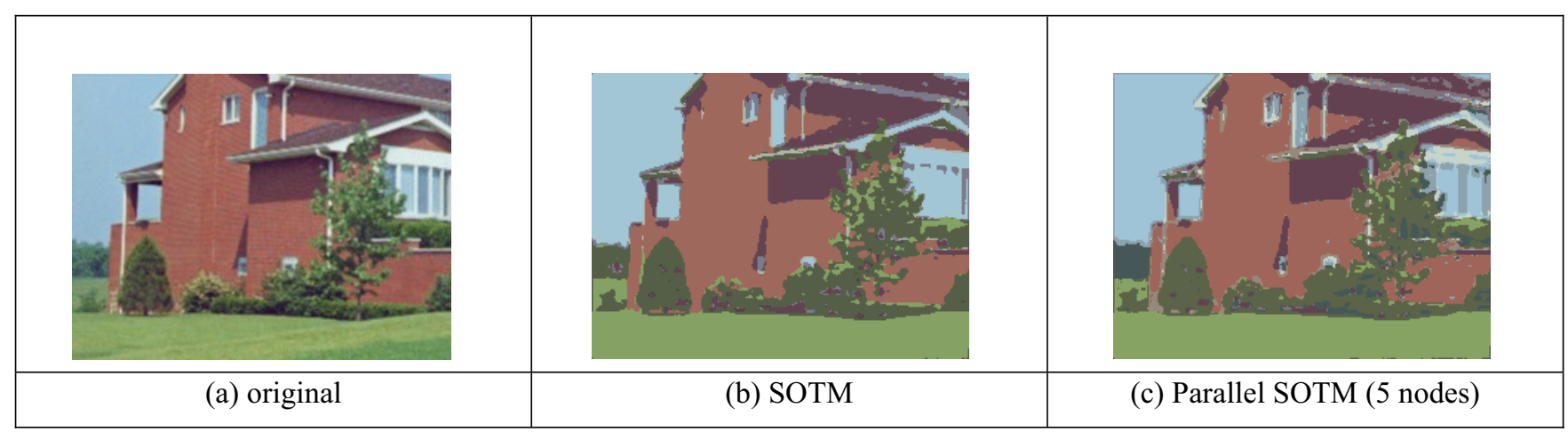

Figure 3 Segmentation results for the House image

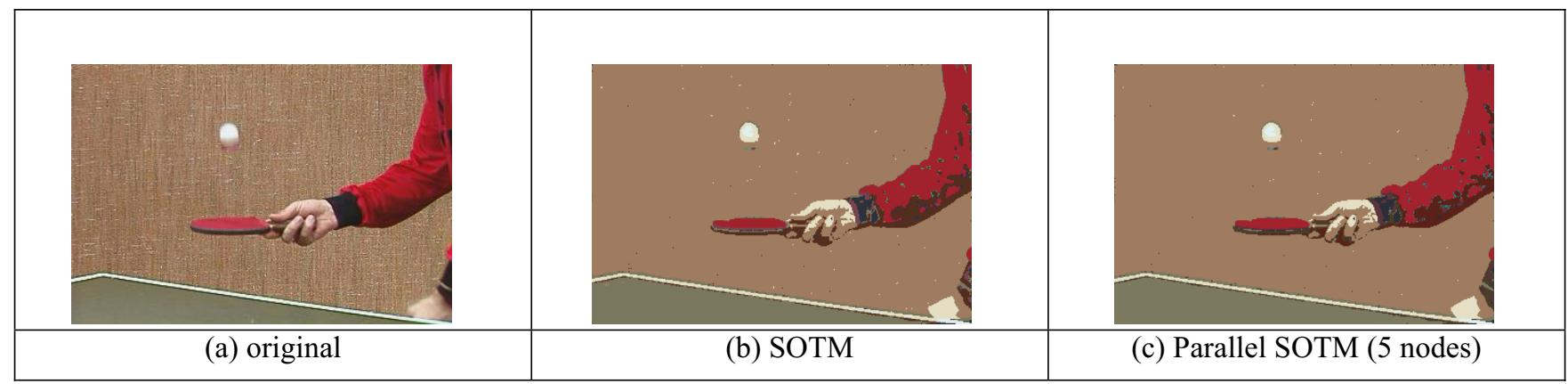

Figure 4 Segmentation results for the PingPong image

process the image of taxing project in an acceptable time and affordable cost.

Current restrictions of conventional SOTM-system implementations, such as limited available CPU, memory and process priority could be solved by PSOTM easily. Results of PSOTM case studies indicate that substantial performance improvements strongly depend on the number of processes. To conclude, PSOTM-technology will be very attractive in high performance image segmentation system, since the system performance could be improved significantly by the increasing the number of processes in a fixed configuration system.

\section{CONCLUSIONS}

By the previous research, we detect that self-organizing tree map is a useful tool in human level image segmentation. In the paper, we show the high efficiency of PSTOM which performs unsupervised learning in parallel.

Potential performance improvement of the image processing system for employing PSOTM has been investigated in the paper. In comparison with SOTM, PSOTM offers the chance to greatly improve the performance of SOTM in image segmentation. By integrating several SOTMs into one system, the PSOTM could fully use the resource of multiple computers to reduce of overall computation time.

From the experimental results, we conclude that PSOTM is capable of improving the computational speed without a severe impact on the performance for image segmentation applications.

\section{REFERENCES}

[1] Y.Deng, and B.S.Manjunath, "Unsupervised segmentation of color-texture regions in images and video," IEEE Transactions on Pattern Analysis and Machine Intelligence (PAMI '01), August 2001.

[2] D. Comaniciu, P. Meer: Robust Analysis of Feature Spaces: Color Image Segmentation, IEEE Conf. Computer Vision and Pattern Recognition (CVPR'97), San Juan, Puerto Rico, 750-755, 1997

[3] Jianbo Shi and Jitendra Malik, "Normalized Cuts and Image Segmentation", IEEE Transactions on pattern analysis and machine intellignce, VOL. 22, NO. 8, August 2000

[4] H. Kong, Self-Organizing Tree Map and Its Applications in Digital Image Processing. PhD thesis, University of Sydney, 1998.

[5] J. Randall and L. Guan, "The hierarchical cluster model for image region segmentation," in Proc. of IEEE International Conference on Multimedia and Expo, pp. 693-696, 2002.

[6] I.T. Jolliffe, Principal Component Analysis, Springer, 2nd edition, 2002. 